\title{
Fatores de risco odontológicos, moleculares e genéticos para o AVC: uma revisão sistemática
}

\author{
Dentistry, molecular, and genetic risk factors for stroke: \\ a systematic review
}
Factores de riesgo dentales, moleculares y genéticos en al ictus: una revisión sistemática
César Antonio Araújo Melo실 ${ }^{1}$ Jullierme de Oliveira Morais ${ }^{2}$, Gabriela de Oliveira Martins ${ }^{3}$, Geórgia Costa de Araújo Souza ${ }^{4}$, Pablo de Castro Santos ${ }^{5}$

\begin{abstract}
1.Acadêmico do curso de Odontologia da Universidade do Estado do Rio Grande do Norte - UERN. CaicóRN, Brasil. ORCID: https://orcid.org/0000-0002-6133-6435

2.Cirurgião Dentista (UERN). Caicó-RN, Brasil. ORCID: https://orcid.org/0000-0003-4410-8681

3. Acadêmica do curso de Odontologia da Universidade do Estado do Rio Grande do Norte - UERN. CaicóRN, Brasil. ORCID: https://orcid.org/0000-0001-6376-0013

4.Cirurgiã-Dentista (UFRN), Doutora em Saúde Coletiva pelo Programa de Pós-graduação em Saúde Coletiva (PPGSCol) da UFRN. Professora do Curso de Odontologia, Departamento de Odontologia da UERN. Caicó-RN, Brasil. ORCID: https://orcid.org/0000-0002-3342-2627

5.Biólogo (UFRN). Doutor em Bioquímica pelo Programa de Pós-graduação em Bioquímica do Centro de Biociências da UFRN. Pós-doutorado em Educação pelo Instituto de Educação da Universidade de Lisboa (IE-UL). Professor do Curso de Odontologia, Departamento de Odontologia da UERN. Professor do Mestrado Profissional em Ensino de Biologia (PROFBIO). Caicó-RN, Brasil. ORCID: https://orcid.org/00000003-0081-9609
\end{abstract}

\begin{abstract}
Resumo
Introdução. As doenças cerebrovasculares são consideradas um problema de saúde pública, dentre elas o Acidente vascular cerebral se destaca pelo seu elevado potencial de causar morbimortalidade. Objetivo. Compreender como os fatores odontológicos, moleculares e genéticos em conjunto potencializam os episódios de Acidente vascular cerebral. Método. $\mathrm{O}$ presente artigo foi estruturado como uma revisão sistemática da literatura e a busca dos artigos foi realizada no período de março a junho de 2020 nos portais da Biblioteca Virtual em Saúde, PubMed e nas bases de dados Scientific Electronic Library Online, ScienceDirect e SpringerLink. Resultados. Pôde-se observar a existência de associação entre a periodontite e Acidente vascular cerebral, também entre as bactérias Actinomycetemcomitans, Staphylococcus, Porphyromonas gingivalis e Streptococcus mutans causadoras de hemorragias mediadas por IL-6 e Acidente vascular cerebral. Já os ácidos graxos como ômega3 e Ácidos Graxos Poli-Insaturados estão associados a prevenção primária e secundária da doença cardiovascular e influenciar no risco de Acidente vascular cerebral. Por fim, alguns estudos sugerem que variações e polimorfismos nos genes BRCA2 e HDAC9 estão associados a doenças cardiovasculares e cerebrovasculares. Conclusão. Destaca-se a importância da contribuição desse conhecimento em conjunto para um melhor prognóstico, segurança do paciente e assistência em saúde.

Unitermos. Transtornos cerebrovasculares; Periodontite; Imunomodulação; Polimorfismo Genético; Streptococcus mutans
\end{abstract}

\footnotetext{
Abstract

Introduction. Cerebrovascular diseases are considered a public health problem, among them the Stroke stands out for its high potential to cause morbidity and mortality. Objective. Understand how the dental, molecular, and genetic factors together enhance the episodes of stroke. Method. This article was structured as a systematic review of the literature and the search for the articles was carried out from March to June 2020 in the portals of the Virtual
} 
Health Library, PubMed and in the Scientific Electronic Library Online, ScienceDirect and SpringerLink databases. Results. It was possible to observe the existence of an association between periodontitis and Stroke, also between the bacteria Actinomycetemcomitans, Staphylococcus, Porphyromonas gingivalis and Streptococcus mutans that cause hemorrhages mediated by IL- 6 and Stroke. Fatty acids such as omega- 3 and polyunsaturated fatty acids are associated with primary and secondary prevention of cardiovascular disease and influence the risk of stroke. Finally, some studies suggest that variations and polymorphisms in the BRCA2 and HDAC9 genes are associated with cardiovascular and cerebrovascular diseases. Conclusion. The importance of the contribution of this knowledge together for a better prognosis, patient safety and health care are highlighted.

Keywords. Cerebrovascular disorders; Periodontitis; Immunomodulation; Genetic polymorphism; Streptococcus mutans

\section{RESUMEN}

Introducción. Las enfermedades cerebrovasculares son consideradas un problema de salud pública, entre ellas el ictus destaca por su alto potencial de causar morbilidad y mortalidad. Objetivo. Comprenda cómo los factores dentales, moleculares y genéticos juntos mejoran los episodios de accidente cerebrovascular. Método. Este artículo se estructuró como una revisión sistemática de la literatura y la búsqueda de los artículos se realizó de marzo a junio de 2020 en los portales de la Biblioteca Virtual en Salud, PubMed y en las bases de datos Scientific Electronic Library Online, ScienceDirect y SpringerLink. Resultados. Se pudo observar la existencia de una asociación entre periodontitis y Stroke, también entre las bacterias Actinomycetemcomitans, Staphylococcus, Porphyromonas gingivalis y Streptococcus mutans que causan hemorragias mediadas por IL-6 y Stroke. Los ácidos grasos como el omega-3 y los ácidos grasos poliinsaturados están asociados con la prevención primaria y secundaria de enfermedades cardiovasculares e influyen en el riesgo de accidente cerebrovascular. Finalmente, algunos estudios sugieren que las variaciones y polimorfismos en los genes BRCA2 y HDAC9 están asociados con enfermedades cardiovasculares y cerebrovasculares. Conclusión. Se destaca la importancia del aporte de este conocimiento de forma conjunta para un mejor pronóstico, seguridad del paciente y asistencia sanitaria.

Palabras clave: Trastornos Cerebrovasculares; Periodontitis; Inmunomodulación; Polimorfismo Genético; Streptococcus mutans

Trabalho realizado na Universidade do Estado do Rio Grande do Norte - UERN. Caicó-RN, Brasil.

Conflito de interesse: não

Recebido em: 07/02/2021

Aceito em: $26 / 07 / 2021$

Endereço para correspondência: Pablo de Castro Santos. Universidade do Estado do Rio Grande do Norte - UERN. Departamento de Odontologia, Campus Caicó. Av Rio Branco 725. Caicó-RN, Brasil. CEP 59300-000. Telefone (84) 3421-6513; (84) 98879-8880. Email: pablodecastrosantos@gmail.com / pablocastro@uern.br

\section{INTRODUÇÃO}

As doenças cerebrovasculares (DCbVs) são caracterizadas por um desenvolvimento rápido do quadro clínico e sintomático causados por um distúrbio local do cérebro ou sistêmico que pode resultar em morte. Dentre essas DCbVs o acidente vascular cerebral (AVC) se encontra com o maior destaque, dentre outros motivos, pela sua elevada incidência, com 17 milhões de indivíduos acometidos 
anualmente, dentre os quais, 6 milhões evoluem para óbito $^{1,2}$.

O AVC, pode abranger além do cérebro, o encéfalo e ambos são precedidos de desordens ocorridas em qualquer parte ao longo do sistema vascular. Não obstante, algumas anomalias os afetam vasos aumentando a probabilidade de ocorrência de AVC, tais como: placas de ateroma, aumento de calibre, perda de elasticidade e alterações bioquímicas no sangue, como proteinúria, dislipidemia, espécies reativas de oxigênio e outras substâncias tóxicas para as células do tecido, o que implicam direta ou indiretamente em distúrbios cerebrais e encefálicos ${ }^{3}$. Nesse sentido, pesquisas mostraram que mundialmente, através de seus diversos fatores, as doenças cardiovasculares continuam sendo a principal causa da morte ${ }^{2,4}$.

O AVC caracteriza-se como uma insuficiência temporária ou definitiva que é provocada por alteração da circulação sanguínea no encéfalo e pode causar danos em várias regiões do próprio órgão ou em outras do corpo que possam estar relacionadas a região afetada. Ele pode se manifestar de duas formas principais, isquêmico ou hemorrágico e ambos provocam o comprometimento de funções neurológicas. Diversos são os fatores de risco associados ao desenvolvimento do AVC, contudo, encontrase na literatura evidências de uma maior incidência entre a população idosa que faz parte do grupo com maior vulnerabilidade para o $\mathrm{AVC}^{5,6}$. 
O acometimento por AVC isquêmico ocorre devido a obstrução das principais artérias que levam sangue ao encéfalo e causa hipóxia com a redução no suprimento rico em oxigênio $\left(\mathrm{O}_{2}\right)$ e aumento da concentração de dióxido de carbono $\left(\mathrm{CO}_{2}\right)$. Já o hemorrágico ocorre pelo rompimento de uma artéria do encéfalo, seguido de extravasamento sanguíneo para além da barreira hematoencefálica e aumento da pressão intracraniana. É possível observar na literatura que o AVC isquêmico é o mais prevalente, com aproximadamente $88 \%$ dos casos, no entanto, sua mortalidade é em torno de 15 a $20 \%$ menor quando comparado ao hemorrágico 7 .

Diversos aspectos epidemiológicos são conhecidos para o AVC, como incidências, prevalências, índices relacionados a óbitos, no entanto os aspectos moleculares necessitam de uma melhor compreensão e apesar de existirem estudos isolados, os distúrbios cardiovasculares estão, em sua grande maioria, associados ao aumento de lipoproteínas no sangue, evidenciando que, a presença destas é fortemente ligada as doenças coronarianas e ao $\mathrm{AVC}^{8}$. Há também a possibilidade de uma predisposição genética que não pode ser controlada e dessa forma interferir na formação embrionária, nas funções celulares, histológicas, anatômicas vasculares e bioquímicas, importantes para a manutenção estrutural dos vasos ${ }^{9}$.

Já dentre os aspectos comportamentais, pode-se observar que após o acometimento por AVC é comum episódios de depressão e outros problemas psicossociais, 
associados ao aumento da mortalidade e verifica-se ainda, que essa associação é mais forte entre os mais jovens ${ }^{10}$.

Existem também dois estudos que sugerem a relação da condição bucal ao risco de episódio de $\mathrm{AVC}^{11,12}$, principalmente quando associados a doenças infecciosas originadas da cavidade oral. Alguns mecanismos patogênicos orais se dão pela disseminação e propagação de forma sistêmica de microrganismos presentes na cavidade oral, que ao adentrarem na corrente sanguínea, muitas vezes, induzem reações bioquímicas e moleculares ao ponto de formar trombos, tornando favorável a agregação plaquetária em várias regiões do corpo humano ${ }^{5}$.

Diante do exposto, a proposição de uma revisão sistemática torna-se uma importante ferramenta para se compreender como os fatores odontológicos, moleculares e genéticos em conjunto potencializam os episódios de AVC.

\section{MÉTODO}

O presente artigo foi construído com o intuito de se realizar uma revisão sistemática sem metanálise seguindo as recomendações do Preferred Reporting Items for Systematic Reviews and Meta-Analyses (PRISMA) ${ }^{13}$, cuja finalidade é sistematizar os resultados de pesquisas sobre um determinado tema ou área de conhecimento de forma ordenada para facilitar a compreensão e permitir uma melhor associação entre seus conteúdos. Tendo como pergunta norteadora: quais fatores odontológicos, 
moleculares e genéticos podem potencializar episódios de AVC?

A busca para obtenção dos estudos foi realizada no período de março a junho de 2020. As Bases de Dados escolhidas para a pesquisa foram: Scientific Eletronic Library Online (SciELO), ScienceDirect, SpringerLink e nos portais Biblioteca Virtual de Saúde (BVS) e PubMed. Todas as buscas foram realizadas com descritores em português e inglês respectivamente, determinados pelo DeCS (Descritores em Ciências de Saúde) e MeSH (Medical Subject Headings): Acidente vascular cerebral, Stroke, Molecular, Odontologia, Dentistry, Epidemiologia, Epidemiology.

Todos os descritores foram utilizados de forma simultânea durante a pesquisa nas bases de dados utilizadas, por meio do operador booleano lógico "AND" com o intuito de angariar estudos que contemplassem a maior parte dos temas de forma combinada para poder se responder à pergunta e fazer parte do presente estudo. Os critérios de inclusão foram: artigos originais, artigos nas línguas portuguesa e inglesa, artigos de revisão de literatura pertinente ao tema como também estudos experimentais e envolvendo o público dos últimos 10 anos. Foram excluídos estudos nas demais línguas, capítulos de livros, teses e dissertações.

Após a coleta de dados os artigos foram analisados e separados de acordo com a relevância para o tema, em seguida formou-se o contexto para discussão do presente trabalho e foram apresentados os dados por meio de texto 
narrativo. Foram selecionados os artigos que se enquadraram nos critérios de inclusão sendo eles: artigos em português, inglês e espanhol, publicados nos últimos dez anos, completos, conforme fluxograma apresentado na Figura 1.

Figura 1. Fluxograma da seleção dos artigos incluídos na revisão sistemática por base de dados.

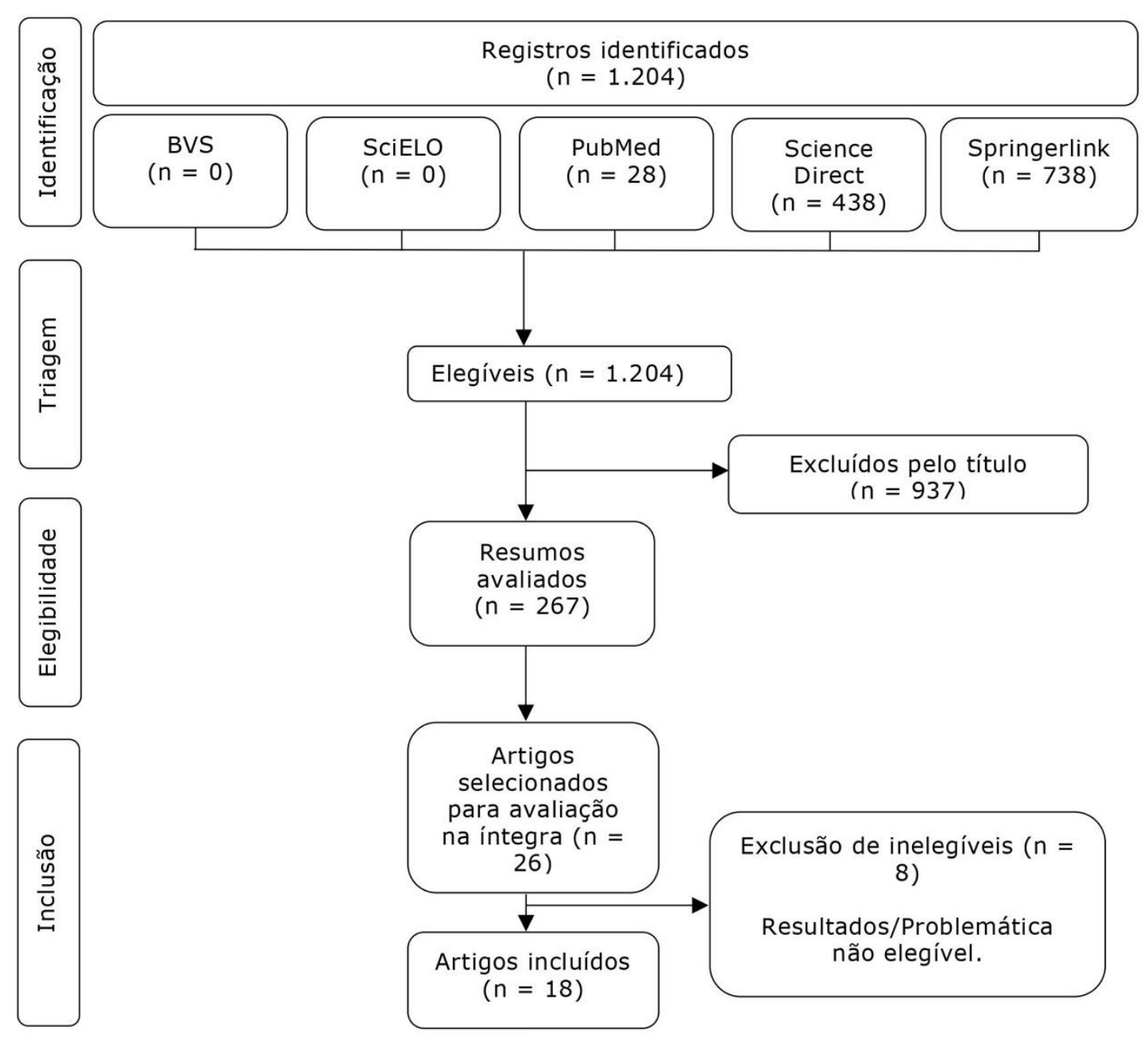

É importante ressaltar que este estudo por se tratar de uma revisão de literatura sistemática não envolve a obtenção de informações diretas ou indiretas de seres humanos e de acordo com as resoluções éticas vigentes, 466/12, 510/16 e 
suas complementares, portanto não necessita de avaliação por um Comitê de Ética em Pesquisa.

\section{RESULTADOS}

O presente estudo contou com uma amostra de 18 artigos publicados entre 2010 e 2020 , cujos resultados foram apresentados conforme as tabelas 1, 2 e 3 considerando em cada tabela o título do artigo analisado, autor, ano de publicação, base de dados que contém o artigo, resultados e conclusões.

Na Tabela 1, foram descritos os estudos que abordam a relação da Odontologia com o AVC. Observou-se que fatores como perda dentária ${ }^{14}$ e doença periodontal causam aumento da carga arteriosclerótica carotídea induzidas por bactérias periodontopatogênicas, como $A$. actinomycetemcomitans e $P$. gingivalis e bactérias da cárie dentária como Streptococcus mutans ${ }^{11,15,16}$.

Ao se observar a Tabela 2 é possível perceber como alguns fatores moleculares e genéticos podem estar associados ao acidente vascular cerebral, por exemplo a utilização de óleos ômega $3^{17}$ e ácidos graxos poliinsaturados ${ }^{18}$, segundo os artigos selecionados, podem reduzir a mortalidade em pacientes acometidos por AVC.

Algumas variações no gene HDAC9 ${ }^{9}$ parecem estar associadas a episódios de AVC em vasos calibrosos. Polimorfismos em genes BRCA ${ }^{19}$ podem estar associados a menor risco de doenças cardiovasculares, um fator para predisposição ao AVC, além de outros estudos com 
Tabela 1. Fatores de risco odontológicos para o AVC.

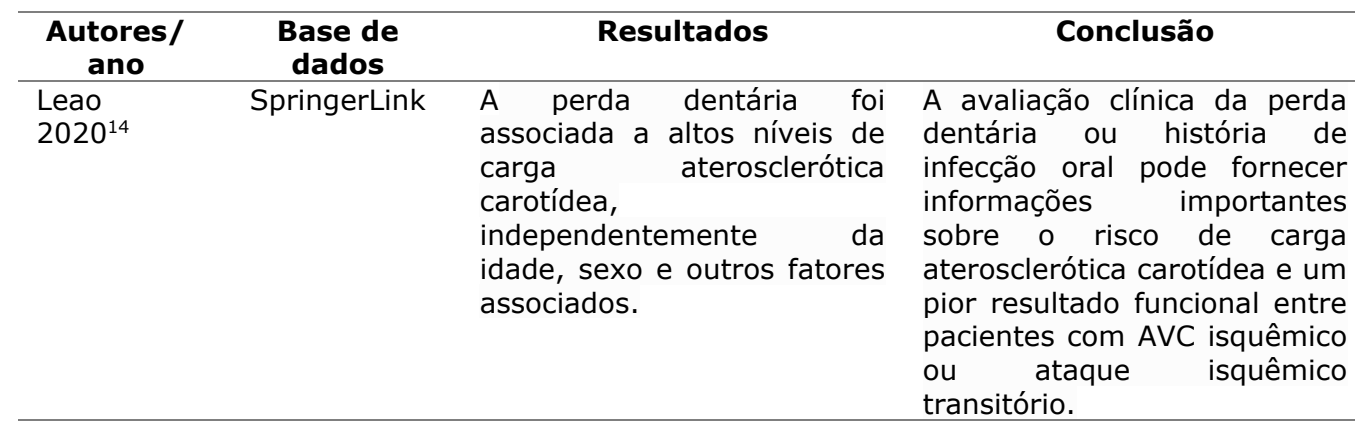

Kebschull PubMed As evidências de estudos Muitos experimentos in vitro e

$2010^{12} \quad$ epidemiológicos sugerem
que as infecções periodontais estão independentemente associadas à doença vascular aterosclerótica subclínica e clínica. Os dados desses estudos contribuem para determinar se as intervenções periodontais têm um papel na prevenção primária ou secundária da aterosclerose.

estudos in vivo estabeleceram a plausibilidade de uma ligação entre infecções periodontais e aterogênese, identificando vias biológicas pelas quais esses efeitos são mediados.

Murakami PubMed Demonstrou-se que os

pacientes com nefropatia diabética tinham mais $A$. actinomycetemcomitans em comparação com nefropatia não diabética na placa dentária. Além disso, os pacientes com nefropatia diabética apresentaram incidência maior de infarto cerebral em comparação com aqueles com nefropatia não diabética.

Ghizoni PubMed O grupo de teste mostrou um $2012^{15}$ aumento significativo em cada um dos seguintes parâmetros: profundidade da bolsa, perda de inserção clínica, sangramento na sondagem, índice de placa e número de dentes quando comparados aos valores de controle $(p<0,05$, teste $t$ não pareado).

Patógenos periodontais,
particularmente
actinomycetemcomitans,
podem desempenhar um
papel, pelo menos uma parte,
no desenvolvimento de infarto
cerebral em pacientes
japoneses em hemodialise
com nefropatia diabética.

Os pacientes com AVC apresentavam bolsas mais profundas, perda de inserção mais severa, aumento do sangramento no aumento dos índices de placa bacteriana e, nos bolsos, aumentavam os níveis de $P$. gingivalis. Esses achados sugerem que a doença periodontal é um fator de risco para o desenvolvimento de hemorragia cerebral ou infarto.

\begin{tabular}{|c|c|c|}
\hline $\begin{array}{l}\text { Beck } \\
2017^{25}\end{array}$ & PubMed & $\begin{array}{l}\text { No estudo a comunidade com } \\
\text { risco aterosclerose a alta } \\
\text { inflamação gengival, perda } \\
\text { dentária, perda dentária } \\
\text { grave, e doença grave do } \\
\text { perfil periodontal foram } \\
\text { significativamente }\end{array}$ \\
\hline
\end{tabular}
associados ao diabetes, doença arterial coronariana (DAC), proteína $C$ reativa de alta sensibilidade e interleucina (IL-6), enquanto apenas doença grave do perfil foi associada ao AVC.
A adição do perfil fenotípico periodontal às variáveis tradicionais de associação de doenças como diabetes, acidente vascular cerebral, doença coronariana forneceu uma clareza maior sobre como o fenótipo pode se referir a essas doenças sistêmicas. 
Tabela 1 (cont.). Fatores de risco odontológicos para o AVC.

\begin{tabular}{|c|c|c|c|}
\hline $\begin{array}{c}\text { Autores/ } \\
\text { ano }\end{array}$ & $\begin{array}{c}\text { Base de } \\
\text { dados }\end{array}$ & Resultados & Conclusão \\
\hline $\begin{array}{l}\text { Cecon } \\
2010^{28}\end{array}$ & Science direct & $\begin{array}{l}\text { Em um grupo testado com } \\
\text { nove pacientes, a contagem } \\
\text { total de bactérias viáveis, } \\
\text { estafilococos, } \\
\text { Enterobacteriaceae e as } \\
\text { leveduras aumentaram } \\
\text { progressivamente de } \\
\text { maneira dependente do } \\
\text { tempo. Dos } 21 \text { casos, dois } \\
\text { em que o AVC levou ao } \\
\text { coma, observados pelo } \\
\text { estudo, os pacientes vieram } \\
\text { a óbito por pneumonia. }\end{array}$ & $\begin{array}{l}\text { Nos pacientes em estado de } \\
\text { coma que tiveram a sua } \\
\text { escovação oral cessada, houve } \\
\text { crescimento progressivo } \\
\text { dessas bactérias podendo } \\
\text { disseminar-se para os } \\
\text { pulmões. }\end{array}$ \\
\hline $\begin{array}{l}\text { Kim } \\
2010^{26}\end{array}$ & PubMed & $\begin{array}{l}\text { A periodontite com nível de } \\
\text { inserção clínica } \geq 6 \mathrm{~mm} \\
\text { mostrou-se } \\
\text { significativamente associada } \\
\text { ao AVC hemorrágico. A } \\
\text { associação entre } \\
\text { periodontite com nível de } \\
\text { inserção clínica } \geq 6 \mathrm{~mm} \text { e AVC } \\
\text { hemorrágico foi significante } \\
\text { em homens, pacientes com } \\
\text { baixa renda, obesos e sem } \\
\text { diabetes. }\end{array}$ & $\begin{array}{l}\text { Periodontite pode ser um fator } \\
\text { de risco independente para } \\
\text { acidente vascular cerebral } \\
\text { hemorrágico. Grupos de risco } \\
\text { incluem homens, pacientes } \\
\text { sem diabetes e obesos. }\end{array}$ \\
\hline $\begin{array}{l}\text { Tonomura } \\
2016^{16}\end{array}$ & PubMed & $\begin{array}{l}\text { A presença de Streptococcus } \\
\text { mutans cnm-positivo foi } \\
\text { significativamente associada } \\
\text { à indução de hemorragia } \\
\text { intracerebral hipertensiva e } \\
\text { número aumentado de micro } \\
\text { sangramentos cerebrais } \\
\text { profundos. Em indivíduos } \\
\text { positivos para Streptococcus } \\
\text { mutans, a atividade de } \\
\text { ligação ao colágeno foi } \\
\text { positivamente } \\
\text { correlacionada com o } \\
\text { número de micro } \\
\text { sangramentos cerebrais. }\end{array}$ & $\begin{array}{l}\text { Esses resultados fornecem } \\
\text { mais evidências sobre a } \\
\text { Streptococcus mutans a } \\
\text { associação ao AVC. }\end{array}$ \\
\hline $\begin{array}{l}\text { Bokhari } \\
2012^{23}\end{array}$ & Science Direct & $\begin{array}{l}\text { Novecentos e trinta e seis } \\
\text { pacientes com doença } \\
\text { arterial coronariana e } 595 \\
\text { indivíduos saudáveis com } \\
\text { idade média de } 51,9 \pm 8,4 \\
\text { anos. A perda dentária média } \\
\text { foi significantemente maior } \\
\text { em pacientes cardíacos. } \\
\text { Após o ajuste da idade, sexo, } \\
\text { diabetes e tabagismo, os } \\
\text { indivíduos com doença } \\
\text { arterial coronariana } \\
\text { apresentaram maior } \\
\text { probabilidade de ter maior } \\
\text { perda dentária. }\end{array}$ & $\begin{array}{l}\text { A perda dentária foi } \\
\text { significativamente associada à } \\
\text { prevalência de doença arterial } \\
\text { coronariana prevalecendo } \\
\text { os fatores de risco clássicos } \\
\text { como: idade, sexo, tabagismo } \\
\text { e diabetes nesta amostra de } \\
\text { estudo. }\end{array}$ \\
\hline
\end{tabular}


Tabela 2. Fatores de risco moleculares e genéticos para o AVC.

\begin{tabular}{ccccccc}
$\begin{array}{c}\text { Autores/ } \\
\text { ano }\end{array}$ & $\begin{array}{c}\text { Base de } \\
\text { dados }\end{array}$ & \multicolumn{3}{c}{ Resultados } & Conclusão \\
\hline Cao & PubMed & Ao final de 1 ano de $\mathrm{Em}$ & conclusão & fica
\end{tabular}

$\begin{array}{llll}\text { Cao } & \text { PubMed Ao final de } & 1 & \text { ano de } \\ 2014^{17} & & \text { acompanhamento, pacientes que }\end{array}$

acompanhamento, pacientes que tomaram o suplemento de óleo de peixe tiveram uma redução de $15 \%$ da morbilidade primária, incluindo 21 e $30 \%$ reduções na mortalidade total cérebro vascular.

Abdelhamid PubMed $\mathrm{O}$ aumento do ácidos graxos poli$2018^{18} \quad$ insaturados (AGPI) provavelmente reduz risco de eventos coronarianos de $14,2 \%$ a $12,3 \%$, em 1.351 pessoas com eventos de doença coronária, 15 ensaios clínicos, evidência de qualidade moderada, imprecisão e risco de viés combinado e acidente vascular cerebral de $1,2 \%$ para $1,1 \%$ em 166 casos e 11 ensaios clínicos, no entanto, os intervalos de confidencialidade incluíam danos importantes, evidência de baixa qualidade rebaixada uma vez por imprecisão e uma vez risco de viés e imprecisão combinados.

Ogorodnikova PubMed A distribuição de casos de acidente $2010^{24} \quad$ vascular cerebral incidente com alto peso molecular por quartis de adiponectina gerou $\mathrm{P}=0,96$ para associação entre diabetes tipo 2, hipertensão, índice de massa corporal alto, glicose, insulina e colesterol. A Razão de probabilidade de acidente vascular cerebral ajustados as multivariáveis associadas aos 3 principais quartis de adiponectina de alto peso molecular versus o primeiro o quartil, gerou $(P=0,14)$.

Bellenguez PubMed O estudo propôs uma nova associação
$2012^{9} \quad$ para episódios de acidente vascular cerebral em vasos calibrosos e variações no gene HDAC9, relacionado a codificação da enzima histona desacetilase 9 no cromossomo 7p21.1.

Sabater-lleal Pubmed Foi conduzida uma meta-análise $2013^{30}$ incluindo 28 estudos com associação entre diferentes genomas. Percebeu-se associação de polimorfismo de nucleotídeo simples com desfechos clínicos em um total de 40.695 casos e 85.582 controles para doença arterial coronariana (DAC), 4.752 casos e 24.030 controles para acidente vascular cerebral e 3.208 casos e 46.167 controles para tromboembolismo venoso (TEV). De forma geral foram observadas variações genéticas responsáveis por alterações do fibrinogênio plasmático e implicações nas e vias relacionadas à inflamação, adipocitocinas e sinalização do hormônio liberador de tireotrofina.
Em conclusão, fica como graxos de ômega-3.

O aumento da ingestão de AGPI pode reduzir levemente o risco de mortalidade por doença cardíaca coronariana e acidente vascular cerebral (embora intervalos de confiança incluem danos importantes), mas podem ter pouco ou nenhum efeito na mortalidade por doenças cardiovasculares (todas de baixa qualidade de evidência).

Não foram encontramos nenhuma evidência de associação entre os níveis de adiponectina de alto peso molecular e acidente vascular cerebral isquêmico nessas mulheres pós-menopáusicas.

Ficou concluído que os quatro locos exibiram evidências de heterogeneidade de efeito nos subtipos de acidente vascular cerebral sugerindo que sequências genéticas distintas para diferentes subtipos de acidente vascular cerebral.

Em análise dos resultados os

loci não suportam uma relação causal entre os níveis circulantes de fibrinogênio e doença arterial coronariana, acidente vascular cerebral ou tromboembolismo venoso. 
Tabela 2 (cont.). Fatores de risco moleculares e genéticos para o AVC.

\begin{tabular}{|c|c|c|c|}
\hline $\begin{array}{c}\text { Autores/ } \\
\text { ano }\end{array}$ & $\begin{array}{c}\text { Base de } \\
\text { dados }\end{array}$ & Resultados & Conclusão \\
\hline $\begin{array}{l}\text { Zbuk } \\
2012^{19}\end{array}$ & $\begin{array}{c}\text { Springerli } \\
\mathrm{nk}\end{array}$ & $\begin{array}{l}\text { Dois polimorfismos de nucleotídeos } \\
\text { únicos (SNPs), rs11571836 e } \\
\text { rs1799943 do gene BRCA2, } \\
\text { localizados em regiões não traduzidas } \\
\text { foram associados ao menor risco de } \\
\text { Doença Cardiovascular (DCV) (OR } \\
0,47 \mathrm{p}=0,01 \text { e OR 0,56 } \mathrm{p}=0,03, \\
\text { respectivamente). Análises em etnias } \\
\text { específicas demonstraram associação } \\
\text { com DCV para ambos os } \\
\text { polimorfismos em população } \\
\text { aborígine, e para rs11571836 apenas } \\
\text { em Sul-asiáticos. E não foi observada } \\
\text { associação nos subgrupos europeu e } \\
\text { chinês. }\end{array}$ & $\begin{array}{l}\text { Embora tenha havido uma associação } \\
\text { entre dois SNPs no gene BRCA2 e } \\
\text { doença cardiovascular em uma } \\
\text { população multiétnica, esses } \\
\text { resultados não foram replicados em } \\
\text { grande número em outras populações }\end{array}$ \\
\hline $\begin{array}{l}\text { Ehret } \\
2011^{20}\end{array}$ & PubMed & $\begin{array}{l}\text { Um escore de risco genético baseado } \\
\text { em } 29 \text { variantes significativas do } \\
\text { genoma foi associado a hipertensão, a } \\
\text { espessura da parede ventricular } \\
\text { esquerda, ao AVC e doença arterial } \\
\text { coronariana. Também observamos } \\
\text { associações com a pressão arterial em } \\
\text { indivíduos com ancestrais do Leste } \\
\text { Asiático, do Sul da Ásia e da África. }\end{array}$ & $\begin{array}{l}\text { As descobertas fornecem novas ideias } \\
\text { sobre a genética e biologia da pressão } \\
\text { arterial e sugerem possíveis novas } \\
\text { vias terapêuticas para prevenção de } \\
\text { doenças cardiovasculares. }\end{array}$ \\
\hline $\begin{array}{l}\text { Sacco } \\
2017^{21}\end{array}$ & PubMed & $\begin{array}{l}\text { No geral, a qualidade das evidências } \\
\text { atuais sobre o risco de acidente } \\
\text { vascular cerebral isquêmico em } \\
\text { pacientes com enxaqueca associado } \\
\text { ao uso de contraceptivos hormonais } \\
\text { foi baixa. Os achados disponíveis } \\
\text { sugerem que o contraceptivo } \\
\text { hormonal combinado pode aumentar } \\
\text { o risco de acidente vascular cerebral } \\
\text { isquêmico em mulheres que têm } \\
\text { enxaqueca, especificamente } \\
\text { enxaqueca com aura, no entanto } \\
\text { existem poucos dados na literatura } \\
\text { para se comprovar. }\end{array}$ & $\begin{array}{l}\text { Para tentar trazer mais segurança e } \\
\text { evitar possíveis riscos ao paciente, } \\
\text { compreende-se que são necessários } \\
\text { mais dados sobre a temática para } \\
\text { maior uso seguro de contraceptivos } \\
\text { hormonais em mulheres com } \\
\text { enxaqueca. }\end{array}$ \\
\hline $\begin{array}{l}\text { Chiappelli } \\
2015^{22}\end{array}$ & $\begin{array}{l}\text { SpringerL } \\
\text { ink }\end{array}$ & $\begin{array}{l}\text { Ebola produz defeitos de coagulação } \\
\text { por produção de citocinas que } \\
\text { diretamente influenciam a cascata de } \\
\text { coagulação. IL1ß, IL2, IL6 e o TNF-a } \\
\text { foram diretamente implicados na } \\
\text { estimulação do sistema de } \\
\text { coagulação. Anormalidades do } \\
\text { cérebro no sistema de coagulação } \\
\text { foram implicados em Lesões } \\
\text { Cerebrais Traumáticas }\end{array}$ & $\begin{array}{l}\text { Essas citocinas têm efeitos } \\
\text { aterogênicos e pró trombóticos, que } \\
\text { podem influenciar diretamente } \\
\text { eventos neurológicos como acidente } \\
\text { vascular cerebral isquêmico ou } \\
\text { demência vascular. }\end{array}$ \\
\hline
\end{tabular}


variantes genéticas ${ }^{20}$ que podem melhorar a compreensão biológica da pressão arterial e sugerir possíveis novas vias terapêuticas. Não obstante foi possível observar que contraceptivos hormonais ${ }^{21}$ quando combinados podem aumentar o risco de acidente vascular em mulheres. Também citocinas inflamatórias como as IL1ß, IL2, IL6 e o TNF-a que são exacerbadas pelo organismo durante alguma doença como o Ebola, a periodontite e outras moduladoras do sistema imunológico, podem estimular a cascata de coagulação, promover uma hipercoagulação ${ }^{22}$ impactando no AVC, incluindo também fatores sistêmicos ${ }^{23}$.

As características demográficas de 9 artigos, em que foi utilizada a participação de pessoas nos estudos, podem ser visualizadas na Tabela 3 . O número de indivíduos nos estudos variou de pelo menos 21 a no máximo 15.792 participantes. A média de idade dos indivíduos que participaram dos estudos variou de 20 a, no máximo, 96 anos.

\section{DISCUSSÃO}

Dentre todos os trabalhos apresentados nas duas tabelas, o acidente vascular isquêmico foi apontado por seis $\operatorname{artigos}^{6,14,16,21,23,24}$, os quais mostraram associação de pessoas que possuem enxaquecas e mesmo tipo de acidente ${ }^{15,21}$, bem como indivíduos que possuem bolsas periodontais e perda de inserção do ligamento periodontal ${ }^{11,12,15,25}$. Três estudos correlacionaram de forma positiva a periodontite e o AVC Hemorrágico ${ }^{15,16,26}$. 
Tabela 3. Dados demográficos dos participantes dos estudos.

\begin{tabular}{|c|c|c|c|c|c|}
\hline Autores & $\begin{array}{l}\text { No de } \\
\text { participantes }\end{array}$ & Sexo & Idade & Local & Doenças associadas \\
\hline Leao $^{14}$ & 459 & M & 20 a 96 & Brasil & $\begin{array}{l}\text { Hipertensão, Diabetes } \\
\text { Mellitus, Aterosclerose, } \\
\text { AVC }\end{array}$ \\
\hline Murakami $^{11}$ & 21 & $\mathrm{M}$ e $\mathrm{F}$ & 40 a 80 & Japão & $\begin{array}{l}\text { Diabetes Mellitus, Doença } \\
\text { renal, } \\
\text { bacteriana, AVC Infecção }\end{array}$ \\
\hline Ghizoni $^{15}$ & 80 & $\mathrm{M}$ e $\mathrm{F}$ & 30 a 80 & Brasil & $\begin{array}{l}\text { Infecção bacteriana oral, } \\
\text { AVC }\end{array}$ \\
\hline Beck $^{25}$ & 15.792 & $\mathrm{MeF}$ & 45 a 64 & Estados Unidos & $\begin{array}{l}\text { Diabetes Mellitus, } \\
\text { Aterosclerose, AVC }\end{array}$ \\
\hline Cecon $^{28}$ & 21 & $\mathrm{M}$ e $\mathrm{F}$ & 22 a 77 & Brasil & $\begin{array}{l}\text { Infecção bacteriana e } \\
\text { fúngica oral, AVC }\end{array}$ \\
\hline $\mathrm{Kim}^{26}$ & 379 & $\mathrm{M}$ e $\mathrm{F}$ & 40 a 79 & Estados Unidos & $\begin{array}{l}\text { Diabetes Melitus, } \\
\text { Hipertensão, AVC }\end{array}$ \\
\hline Tonomura $^{16}$ & 99 & $\mathrm{Me} \mathrm{F}$ & 57 a 83 & Reino Unido & $\begin{array}{l}\text { Hipertensão, } \\
\text { cardiopatias, AVC }\end{array}$ \\
\hline Bokhari ${ }^{23}$ & 1.531 & $\mathrm{MeF}$ & 40 a 70 & França & $\begin{array}{l}\text { Diabetes Melitus } \\
\text { cardiopatias, AVC }\end{array}$ \\
\hline Ogorodnikova ${ }^{24}$ & 1944 & $\mathrm{~F}$ & 50 a 59 & Estados Unidos & Diabetes Melitus, AVC \\
\hline
\end{tabular}

Como foi citada nas doenças associadas, na Tabela 3, é possível destacar como a Diabetes Mellitus (DM) associa-se diretamente com as doenças cerebrovasculares ${ }^{11,23-26}$ uma vez que a hiperglicemia - alto nível de glicose no sangue que proporciona complicações crônicas de diabetes - provoca alterações os tecidos vasculares e, proporcionam a formação de aterosclerose ${ }^{14}$.

As alterações vasculares causadas pela DM crônica podem ser microvasculares ou macrovasculares ${ }^{14,23}$. A complicação crônica microvascular causa a obstrução dos pequenos vasos sanguíneos, como capilares e arteríolas ${ }^{3}$. Os órgãos de maior acometimento dessa patologia são retina e rins, no entanto, outros tecidos podem ser afetados em menor grau ${ }^{11}$. A macrovascular crônica causa obstrução dos grandes vasos, em que suas complicações se aparentam com uma aterosclerose grave ${ }^{14,16,25,26}$. Essa formação ocorre por 
causa do alto índice de glicose interagindo com lipoproteínas e proteínas encontradas no sangue, que vão se depositando nas paredes dos vasos, obstruindo a passagem sanguínea. Assim, ao obstruir o fluxo sanguíneo para o cérebro, o AVC pode ocorrer $16,23,25,26$.

$\mathrm{Na}$ análise dos dados foi encontrado que a periodontite foi um fator preditivo para o AVC, com destaque para os achados: bolsa de profundidade, perda de inserção clínica, sangramento na sondagem, índice de placa e número de dentes quando comparados aos valores de controle ${ }^{11,14,25}$. Dessa forma, todos estes fatores têm contribuído para uma associação positiva com o AVC. Esses achados corroboram com os estudos de $\mathrm{Sen}^{27}$, mostrando que a periodontite grave tem associação significativa com eventos vasculares recorrentes e aumento da espessura da placa do ateroma do arco aórtico.

Nos artigos selecionados foi verificado que a presença de alguns grupos de bactérias na corrente sanguínea tem trazido prejuízos e danos ao sistema cardiovascular, podendo consequentemente ocorrer episódios de AVC. Tal mecanismo é mostrado em estudos de Kebschull ${ }^{12}$, no qual muitas dessas bactérias disseminam-se para a corrente sanguínea através do rompimento de vasos do periodonto, por ser um tecido em sua maioria conjuntivo, como é caso das bactérias Actinomycetemcomitans, Staphylococcus e Porphyromonas gingivalis presentes nas bolsas periodontais ${ }^{11,15,28}$. 
Outro microrganismo importante é o Streptococcus mutans, muito presente na superfície dos dentes, que tem sua penetração no sangue pelo tecido pulpar através da cárie, levando a vários problemas vasculares, causando hemorragias e micro hemorragias cerebrais ${ }^{16}$. Tais sangramentos são extremamente prejudiciais na região cerebral por terem componentes tóxicos no sangue que vão para esse tecido. Esses são precedidos de respostas inflamatórias mediadas por IL-6, evidenciadas em altas quantidades, encontradas em ateroscleroses causadas por doenças moduladoras do sistema imunológico como as periodontais, observado por Beck ${ }^{25}$ e Ebola por Chiappelli22, que tem caráter de provocar defeitos na coagulação sanguínea, culminando em surgimento e agravamento de casos de AVC.

Alguns fatores moleculares encontrados indicam que ácidos graxos como ômega-3 estão associados à prevenção primária e secundária da doença cardiovascular. Foi observado que pacientes que tomaram o suplemento de óleo de peixe tiveram uma redução de $15 \%$ morbilidade primária, incluindo 21 e $30 \%$ de reduções na mortalidade total cérebro vascular ${ }^{17}$. Enquanto em outro estudo, feito de forma sistemática ${ }^{18}$, aponta que a ingestão de AGPI pode diminuir levemente o risco de AVC e tem efeitos ínfimos na questão de mortalidade de DCbV.

Doenças coronárias estão, muitas vezes, relacionadas diretamente com DCbV causadoras de $\mathrm{AVCs}^{2,19,23}$. Em um artigo ${ }^{14}$, não se observou associação entre a perda dentária 
e quadros de AVC isquêmico e isquêmico transitórios, no entanto levaram a uma maior comorbidade após o acidente. Porém em outros artigos de Bokhari ${ }^{23}$ e Ghizoni ${ }^{21}$ foi observado que a ausência de elementos dentários constituiu um fator relacionado ao AVC, uma vez que a perda dentária pode ser reflexo de problemas vasculares, doenças gengivais e estarem relacionadas as DCV e acidentes vasculares.

Alguns dados indicam que o uso de contraceptivos hormonais femininos combinados pode aumentar o risco de acidente vascular cerebral isquêmico em indivíduos que têm enxaqueca, principalmente os com aura ${ }^{21}$. Esse achado corrobora com o estudo de Androulakis ${ }^{29}$ e Ghizoni ${ }^{15}$, que sugerem um risco aumentado de acidente vascular cerebral isquêmico associado à enxaqueca com aura. Contudo, mais estudos são necessários para ter um desfecho plausível diante dessa associação.

Foi observado um crescimento de AVCs primários e recorrentes em jovens abaixo da idade de 50 anos, em especial na faixa de 18 a 34 anos $^{10}$. Corroborando ao achado, Ghizoni ${ }^{15}$ aponta que $32 \%$ de 141 pacientes de outro estudo incluído por ela se encontravam dentro da categoria de jovens, o que é um número alto. Entretanto, considerando a média de idade em seu próprio trabalho, somente duas pessoas eram abaixo de 40 anos.

No campo da genética molecular, um escore de risco genético baseado em 29 variantes genômicas mostrou significativa associação com hipertensão, acidente vascular cerebral e doença arterial coronariana ${ }^{20}$. Por fim, outros 
estudos ainda sugerem que variações e polimorfismos gênicos como os do BRCA2 e HDAC9 podem apontar importantes descobertas para a genéticas e biologia vascular e abrem possibilidades para se testar e compreender novas vias terapêuticas para prevenção de doenças cardiovasculares e cerebrovasculares como o $\mathrm{AVC}^{9,19}$.

\section{CONCLUSÕES}

A condição de saúde bucal associada a investigação de dados moleculares pode fornecer informações importantes relacionadas a predisposição para episódios de Acidente vascular cerebral, com destaque para a presença de microrganismos patogênicos no periodonto que podem provocar alterações na cascata de coagulação, gerando efeitos moleculares e sistêmicos, favorecendo 0 desenvolvimento do AVC. Portanto, observa-se que as doenças periodontais constituem fatores de risco para o AVC.

Ainda, variações e polimorfismos em genes específicos estão relacionados a doenças cardiovasculares e cerebrovasculares e alguns ácidos graxos como ômega-3 e outros AGPI estão associados à prevenção primária e secundária da doença cardiovascular e podem reduzir os riscos de acidente cerebrovascular. Em conjunto a compreensão destes fatores por profissionais capacitados é importante para prevenir episódios de AVC e contribuir para um melhor prognóstico, segurança do paciente e assistência em saúde. Outrossim, destaca-se a necessidade de 
desenvolvimento de mais pesquisas que abordem a relação dos fatores associados ao AVC de forma conjunta.

\section{REFERÊNCIAS}

1.Pannain GD, Ribeiro CC, Jacob MB, Pires LA, Almeida ALM. Relato de experiência: Dia Mundial do Acidente Vascular Cerebral. HU Rev 2019;45:104-8.

https://doi.org/10.34019/1982-

8047.2019.v45.25663

2.Benjamin EJ, Virani SS, Callaway CW, Chamberlain AM, Chang AR, Cheng S, et al. Heart Disease and Stroke Statistics-2017 Update. Circulation 2017;135:146-603.

https://doi.org/10.1161/CIR.0000000000000485

3.Chandra A, Li W, Stone C, Geng X, Ding Y. The cerebral circulation and cerebrovascular disease II: Pathogenesis of cerebrovascular disease. Brain Circ 2017;1:57-65.

https://doi.org/10.4103/bc.bc 1117

4.Gakidou E, Afshin A, Abajobir AA, Abate KH, Abbafati C, Abbas KM, et al. Global, regional, and national comparative risk assessment of 84 behavioural, environmental and occupational, and metabolic risks or clusters of risks, 1990-2016: A systematic analysis for the Global Burden of Disease Study 2016. Lancet 2017;390:1345-422. https://doi.org/10.1016/S0140-6736(17)32366-8

5.Cotti E, Dessì C, Piras A, Mercuro G. Can a chronic dental infection be considered a cause of cardiovascular disease? A review of the literature. Int J Cardiol 2011;148:4-10.

http://dx.doi.org/10.1016/j.ijcard.2010.08.011

6. Costa FA, Silva DLA, da Rocha VM. Severidade clínica e funcionalidade de pacientes hemiplégicos pós-AVC agudo atendidos nos serviços públicos de fisioterapia de Natal (RN). Cienc Saude Col 2011;16(suppl 1):1341-8.

https://doi.org/10.1590/S1413-

81232011000700068

7.Thom T, Haase N, Rosamond W, Howard VJ, Rumsfeld J, Manolio T, et al. Heart disease and stroke statistics - 2006 Update: A report from the American Heart Association Statistics Committee and Stroke Statistics Subcommittee. Circulation 2006;113:85-151. https://doi.org/10.1161/CIRCULATIONAHA.105.171600

8. Danesh J, Collins R, Peto R. Clinical Investigation and Reports MetaAnalysis of Prospective Studies. Circulation 2000;102:1082-5. https://doi.org/10.1161/01.CIR.102.10.1082

9. Bellenguez C, Bevan S, Gschwendtner A, Spencer CCA, Burgess AI, Pirinen $M$, et al. Genome-wide association study identifies a variant in HDAC9 associated with large vessel ischemic stroke. Nat Genet 2012;44:328-33. https://doi.org/10.1038/ng.1081

10. Maaijwee NAMM, Rutten-Jacobs LCA, Schaapsmeerders P, Van Dijk EJ, De Leeuw FE. Ischaemic stroke in young adults: Risk factors and long-term consequences. Nat Rev Neurol 2014;10:315-25. https://doi.org/10.1038/nrneurol.2014.72 
11.Murakami M, Suzuki J, Yamazaki S, Ikezoe M, Matsushima R, Ashigaki $\mathrm{N}$, et al. High incidence of Aggregatibacter actinomycetemcomitans infection in patients with cerebral infarction and diabetic renal failure: A cross-sectional study. BMC Infect Dis 2013;13:557. https://doi.org/10.1186/1471-2334-13-557

12.Kebschull M, Demmer RT, Papapanou PN. "Gum bug, leave my heart alone!" - epidemiologic and mechanistic evidence linking periodontal infections and atherosclerosis. J Dent Res 2010;89:879902. https://doi.org/10.1177/0022034510375281

13. Moher D, Liberati A, Tetzlaff J, Altman DG, Grp P. Preferred Reporting Items for Systematic Reviews and Meta-Analyses: The PRISMA Statement (Reprinted from Annals of Internal Medicine). Phys Ther 2009;89:873-80.

https://doi.org/10.1371/journal.pmed.1000097

14. Leao TS, Tomasi G, Ibrahim MS, Conzatti L, Marrone LP, Reynolds $\mathrm{MA}$, et al. Tooth loss is associated with atherosclerosis and a poorer functional outcome among stroke patients. Clin Oral Investig 2020;24: 4541-8. https://doi.org/10.1007/s00784-020-03319-z

15.Ghizoni JS, Taveira LAA, Garlet GP, Ghizoni MF, Pereira JR, Dionísio $\mathrm{TJ}$, et al. Increased levels of Porphyromonas gingivalis are associated with ischemic and hemorrhagic cerebrovascular disease in humans: an in vivo study. J Appl Oral Sci 2012;20:104-12. https://doi.org/10.1590/S1678-77572012000100019

16. Tonomura S, Ihara M, Kawano T, Tanaka T, Okuno Y, Saito S, et al. Intracerebral hemorrhage and deep microbleeds associated with cnmpositive Streptococcus mutans; A hospital cohort study. Sci Rep 2016;6:1-8. http://dx.doi.org/10.1038/srep20074

17. Cao Y, Lu L, Liang J, Liu M, Li X, Sun RR, et al. Omega-3 Fatty Acids and Primary and Secondary Prevention of Cardiovascular Disease. Cell Biochem Biophys 2015;72:77-81. https://doi.org/10.1007/s12013014-0407-5

18.Abdelhamid A, Martin N, Bridges C, Brainard J, Wang X, Brown T, et al. Polyunsaturated fatty acids for the primary and secondary prevention of cardiovascular disease (Review). Cochrane Database Syst Rev 2018;11:CD012345.

https://doi.org/10.1002/14651858.CD012345.pub3

19.Zbuk K, Xie C, Young R, Heydarpour M, Pare G, Davis AD, et al. BRCA2 Variants and cardiovascular disease in a multi-ethnic study. BMC Med Genet 2012;13:56. https://doi.org/10.1186/1471-2350-13$\underline{56}$

20.Ehret GB, Munroe PB, Rice KM, Bochud M, Johnson AD, Chasman $\mathrm{DI}$, et al. Genetic variants in novel pathways influence blood pressure and cardiovascular disease risk. Nature 2011;478:103-9. http://dx.doi.org/10.1038/nature10405

21.Sacco S, Merki-Feld GS, Ægidius KL, Bitzer J, Canonico M, Kurth T, et al. Hormonal contraceptives and risk of ischemic stroke in women with migraine: a consensus statement from the European Headache Federation (EHF) and the European Society of Contraception and Reproductive Health (ESC). J Headache Pain 2017;18:1-20. 
https://doi.org/10.1186/s10194-017-0815-1

22.Chiappelli F, Bakhordarian A, Thames AD, Du AM, Jan AL, Nahcivan $\mathrm{M}$, et al. Ebola: Translational science considerations. J Transl Med 2015;13:1-29. https://doi.org/10.1186/s12967-014-0362-3

23.Bokhari SAH, Khan AA, Ansari JA, Alam R. Tooth loss in institutionalized coronary heart disease patients of Punjab Institute of Cardiology, Lahore, Pakistan. J Epidemiol Glob Health 2012;2:51-6. http://dx.doi.org/10.1016/j.jegh.2011.11.004

24.Ogorodnikova AD, Wassertheil-Smoller S, Mancuso P, Sowers MR, Rajpathak SN, Allison MA, et al. High-molecular-weight adiponectin and incident ischemic stroke in postmenopausal women: A women's health initiative study. Stroke 2010;41:1376-81. https://doi.org/10.1161/STROKEAHA.109.576546

25.Beck JD, Moss KL, Morelli T, Offenbacher S. Periodontal profile class is associated with prevalent diabetes, coronary heart disease, stroke, and systemic markers of C-reactive protein and interleukin-6. J Periodontol 2018;89:157-65. https://doi.org/10.1002/JPER.17-0426 26.Kim H-D, Sim S-J, Moon J-Y, Hong Y-C, Han D-H. Association Between Periodontitis and Hemorrhagic Stroke Among Koreans: A Case-Control Study. J Periodontol 2010;81:658-65. https://doi.org/10.1902/jop.2010.090614

27.Sen S, Chung M, Duda V, Giamberardino L, Hinderliter A, Offenbacher S. Periodontal Disease Associated with Aortic Arch Atheroma in Patients with Stroke or Transient Ischemic Attack. J Stroke Cerebrovasc Dis 2017;26:2137-44.

http://dx.doi.org/10.1016/j.jstrokecerebrovasdis. 2017.04.035

28.Cecon F, Ferreira LEN, Rosa RT, Gursky LC, de Paula e Carvalho A, Samaranayake LP, et al. Time-related Increase of Staphylococci, Enterobacteriaceae and Yeasts in the Oral Cavities of Comatose Patients. J Microbiol Immunol Infect 2010;43:457-63. http://dx.doi.org/10.1016/S1684-1182(10)60071-0

29.Androulakis XM, Kodumuri N, Giamberardino LD, Rosamond WD, Gottesman RF, Yim E, et al. Ischemic stroke subtypes and migraine with visual aura in the ARIC study. Neurology 2016;87:2527-32. https://doi.org/10.1212/WNL.0000000000003428

30.Sabater-Lleal M, Huang J, Chasman D, Naitza S, Dehghan A, Johnson $A D$, et al. Multiethnic meta-analysis of genome-wide association studies in $>100,000$ subjects identifies 23 fibrinogenassociated loci but no strong evidence of a causal association between circulating fibrinogen and cardiovascular disease. Circulation 2013;128:1310-24.

https://doi.org/10.1161/CIRCULATIONAHA.113.002251 